\title{
Praxisinformationssysteme der Zukunft
}

\author{
Die Unterstützung der administrativen Prozesse einer Arztpraxis durch die In- \\ formatik ist etabliert. Die Zukunft der Praxisinformatik wird durch die Unter- \\ stützung der medizinischen Prozesse geprägt sein. Folgerichtig hat die zu- \\ künftige Ärztesoftware die elektronische Krankengeschichte als zentrales \\ Modul. Die Software muss die Prozesse Dokumentation, Kommunikation und \\ Interaktion unterstützen. Der Klassifizierung kommt inskünftig zentrale Be- \\ deutung zu. Demoversionen und Möglichkeit der Datenmigration sind für die \\ Praxissoftware von morgen zu fordern. Nur diejenigen Anbieter, die die Be- \\ dürfnisse der Ärzte umsetzen, werden überleben.
}

Heinz Bhend,

Leiter AG SGAM.Informatics

Korrespondenz:

Dr. med. Heinz Bhend

Facharzt für Allgemeinmedizin FMH

Informatiker HTA/FHZ

Oltnerstrasse 9

CH-4663 Aarburg

heinz.bhend@sgam.ch
Aus der Administration ist die Computerunterstützung nicht mehr wegzudenken. Hier sind die Prozesse, die computerisiert sind, überblickbar und unbestritten: Patientenadministration, Tarifhandling, Rechnungsstellung, Mahnwesen und meist auch eine Form der Dokumentenverwaltung sind etabliert.

Computerunterstützung bei der eigentlichen Kerntätigkeit des Arztes, d.h. der medizinischen Tätigkeit, ist Neuland in der Praxisinformatik und führt zu Unsicherheiten sowohl bei Anwendern als auch bei Anbietern. Teilweise ist diese Unsicherheit durch Unkenntnis der Möglichkeiten, wohl mehr aber durch die notwendigen Investitionen sowie den unabdingbaren Kulturwandel bedingt. Die mangelnde Austauschbarkeit (infolge fehlender Standards), die Abhängigkeit von Anbietern, der grosse finanzielle Aufwand und der Zeitbedarf für die Umstellung fördern den «going paperless»-Prozess nicht. Im folgenden soll versucht werden, den Status quo und die potentiellen Möglichkeiten der Informatik in der Arztpraxis vorzustellen.

\section{Elektronische KG als zentrales Element}

Die zukünftige Praxissoftware hat als zentrales Modul eine elektronische Krankengeschichte. Anbieter ohne elektronische KG haben im Markt von morgen nichts zu suchen. Diejenigen, die die elektronische KG schon implementiert haben, tun gut daran, das Design ihres Produktes so zu überarbeiten, dass die elektronische KG tatsächlich im Mittelpunkt steht und nicht als «Anhängsel» wahrgenommen wird.

Dies bedeutet, dass die Administration in den Hintergrund rückt [1]. Bisher wurden die administrativen Prozesse informationstechnisch unterstützt bzw. von der Software übernommen. Längst sind aber weitere Prozesse der Arztpraxis einer Informatisierung zugänglich. Der Einsatz der Informatik im Bereich der Medikamentenverwaltung ist nicht nur möglich, sondern auch sinnvoll und ganz konkret qualitätsfördernd [2]. Anhand des Medikamentenmanagements kann illustriert werden, welche Bereiche die Praxissoftware der Zukunft anbieten bzw. abdecken muss. Schon alleine die Übersicht $\mathrm{zu}$ haben über die verschiedenen Formen und Angebote $\mathrm{zu}$ einer bestimmten Substanz ist ohne Informatikunterstützung kaum mehr möglich. Die Möglichkeiten der Informatik kommen aber erst richtig zum Tragen, wenn Interaktionschecks, Unverträglichkeitswarnungen, automatische Preisberechnungen, Dosierungsvorschläge und Berechnung der Behandlungsdauer bei einer bestimmten Originalpackung automatisiert und von der Software zur Verfügung gestellt werden.

\section{Abbilden der Praxisprozesse}

Im Workflow einer Arztpraxis geht es um die Prozesse Dokumentation, Kommunikation und Interaktion. Mit der Papierkrankengeschichte ist nur die Dokumentation möglich. Diese dient lediglich als Gedächtnisstütze und um der gesetzlichen Dokumentationspflicht zu genügen. Mit den heutigen Möglichkeiten der strukturierten, elektronischen Dokumentation bieten sich mit einem geringen Mehraufwand bei der Datenerfassung und dem unabdingbaren Kulturwandel (Computer im Sprechzimmer) derart viele neue Möglichkeiten, dass es beinahe müssig ist, über Sinn oder Unsinn einer elektronischen Krankengeschichte zu debattieren. 
Ein Mehraufwand ist nicht wegzudiskutieren: Die Eingabe erfolgt in der Anfangsphase allenfalls langsamer. Der finanzielle Aufwand und die zusätzlichen Arbeitstunden, bis der Prozess zur papierarmen Praxis einigermassen abgeschlossen ist, sind nicht zu unterschätzen.

Ferner muss und wird ein Kulturwandel stattfinden: Im Sprechzimmer finden sich zusätzlich minimal eine Tastatur und ein Bildschirm.

Befürchtungen einer Störung der Arzt-Patienten-Beziehung werden v.a. von Kolleginnen und Kollegen geäussert, die nie in einem solchen Setting gearbeitet haben, und konnten durch neueste Arbeiten widerlegt werden [3]. Selbstverständlich hängt dies sehr davon $\mathrm{ab}$, wie man den Computer in die Sprechstundensituation integriert, den Patienten informiert und allenfalls miteinbezieht [4].

\section{Strukturierte Dokumentation ...}

Mit der elektronischen Dokumentation ist der Grundstein gelegt, um die patientenspezifischen Informationen in irgendeiner Form, zu einem beliebigen Zeitpunkt und sogar ortsunabhängig zur Verfügung zu haben. Sämtliche E-HealthProzesse, die ganze E-Health-Strategie des Bundes, Ideen über ein elektronisches Patientendossier usw. sind nur sinnvoll, wenn die initiale Dokumentation in der Arztpraxis elektronisch erfolgt. Als Hausärzte sind wir die erste Anlaufstelle im Gesundheitssystem. Um es auch in einem künftigen E-Gesundheitswesen zu sein, tun wir gut daran, uns dem Strukturwandel nicht zu widersetzen, sondern diesen aktiv mitzugestalten.

Die elektronische Dokumentation hat das Potential, nachgelagerte Prozesse zu erleichtern oder erst zu ermöglichen. Sie dient aber primär der eigenen Qualitätsförderung.

$\mathrm{Da}$ es zum Lesen der Informationen keine Interpretationshilfe des Autors mehr braucht (wie so oft bei Papier-KGs), ist die Informationsqualität a priori höher. Informationen können von Dritten (z.B. MPA) bei Bedarf wiedergegeben werden.

Einzelne Teile, ganze Verläufe oder Extrakte können in beliebiger, modularer Form in weitere Dokumente übernommen werden. Konkret bedeutet dies: Aktuelle Medikation, frühere Operationen, Labordaten usw. sind problemlos in Berichte und Zuweisungsschreiben exportierbar.

Anfragen ans System sollten jederzeit möglich sein. Auch hier hat die Ärzteschaft den Lead zu übernehmen. In einer gutgeführten Krankengeschichte sollten jederzeit folgende oder ähnliche Fragen durch die Software beantwortet werden können [5]:
Gesucht werden sämtliche Patienten, die

- ein $\mathrm{Hb}_{\mathrm{A1c}}$ von über 9,0\% haben;

- aktuell ein bestimmtes Medikament (in einer bestimmten Dosierung) verordnet haben;

- ein Statin einnehmen und über 85jährig sind;

- Vorhofflimmern haben, unter 80jährig sind und kein Marcoumar einnehmen.

\section{... ermöglicht zu wissen, was wir tun}

Das Führen einer sinnvollen Problemliste, der Dauer- und Arbeitsdiagnosen oder sogar die Klassifizierung nach ICPC-2 machen eigene Auswertungen möglich. Wahrscheinlich ist dies das grösste Problem der Papier-KG und ein gewaltiges Potential der elektronischen KG: nicht wissen bzw. wissen, was wir tun! Auswertungen, basierend auf Papierkrankengeschichten, sind beinahe ein Ding der Unmöglichkeit und werden somit kaum gemacht; Forschung auf dem Hintergrund elektronischer KGs ist theoretisch auf Knopfdruck möglich [6].

Die elektronische Dokumentation ermöglicht auch die Ablage von Literatur und/oder Guidelines direkt beim Patienten bzw. beim einzelnen Problem.

\section{Weniger Raumbedarf}

Nicht zu unterschätzen ist der Benefit der elektronischen Dokumentation bezüglich Raumbedarfs.

Das Informationsvolumen einer Gruppenpraxis hat problemlos auf einer Festplatte Platz; dies spart somit mehrere Laufmeter an Aktenschränken. Wenn gleichzeitig auch Röntgenfilme, EKGs und Laborblätter digital archiviert werden, ergibt dies ohne weiteres eine Raumersparnis von einem grösseren Arbeitszimmer.

\section{Kommunikation}

Die elektronische Dokumentation ist die Ausgangsbasis sowohl für die interne als auch externe Kommunikation.

\section{Interne Kommunikation}

In einer Praxis mit Papier-KGs wird oft indirekt kommuniziert. So haben beispielsweise gewisse Ablageorte des Dossiers einen Informationsgehalt. Diese Informationen müssen von der Software übernommen werden. Obwohl noch viel Optimierungspotential vorhanden ist, wurde doch schon einiges realisiert: Mitteilungen «auf dem Bildschirm», ohne dass zum Telefon gegriffen werden muss, interne Auftragserteilung, Übersicht der Anzahl Patienten im Wartezimmer usw. sind «state of the art» [7].

Die direkte Geräteanbindung ist ebenfalls Teil der internen Kommunikation. Sie spart Kosten 
(MPA-Power) und verhindert Übertragungsfehler. Röntgen- und Sonographiebilder, EKGs und Lungenfunktionsdokumente sind schneller verfügbar bei entsprechender Integration. Laborgeräte [8] können inzwischen schon bidirektional angebunden werden, d.h., der Arzt kann in der elektronischen KG einen individuellen Laborauftrag definieren und diesen an das Gerät senden. Die MPA wird dann aufgefordert, die entsprechende Probe an Position X zu stellen. Auf diesen Arbeitsschritt folgen weitere vollautomatisch und stellen die MPA wieder frei für andere Aufgaben.

\section{Kommunikation gegen aussen}

Die primäre Kommunikation gegen aussen erfolgt über die E-Mail-Anbindung. Dass dies ein gesichertes System voraussetzt, versteht sich von selbst. Durch die elektronische Übermittlung sind die Daten weiter verwendbar. Wir profitieren sehr von elektronischen Berichten der Konsiliarärzte, können doch diese Informationen (z.B. Diagnoseliste) in der elektronischen KG wieder verwendet werden.

Die Übermittlung von Röntgenbildern an die nächstbehandelnde Instanz ist technisch möglich und wird mit der weiteren Verbreitung der digitalen Röntgenanlagen an Bedeutung gewinnen.

\section{Interaktion schliesst den Kreis}

Dokumentation und Kommunikation vereinfachen die Arbeit unter Umständen wesentlich. Wie so oft im Leben: Eine gewisse Einarbeitungs- und Gewöhnungszeit ist erforderlich. Erst die Möglichkeiten der Interaktion mit der Praxissoftware steigern die medizinische Qualität.

Eine Mischung aus Dokumentation, Kommunikation und Interaktion stellen die Reminderfunktionen dar. Wenn nach einer Koloskopie vorgeschlagen wird, dass der Patient in fünf Jahren eine Kontrollkoloskopie haben sollte, muss diese Information von der Software zeitgerecht zur Verfügung gestellt werden. Derartige zeitgesteuerte Informationen sind mit einer Papier-KG gar nicht möglich. Die Software jedoch kann mich nach Ablauf der fünf Jahre daran erinnern, kann allenfalls automatisch einen Brief an den Patienten generieren usw. Analog können periodische Laborkontrollen durch die Software überwacht werden. Ich kann mich z.B. entscheiden, dass bei allen Diabetespatienten mindestens halbjährlich das $\mathrm{Hb}_{\mathrm{A} 1 \mathrm{c}}$ kontrolliert werden soll. Dies soll voreingestellt werden können. Falls die Zeit von sechs Monaten vorbei ist und kein $\mathrm{Hb}_{\mathrm{A1c}}$-Eintrag erfolgte, meldet sich das System. Falls der Wert gemessen wurde, bleibt es stumm.

Diese Weiterentwicklung der Interaktion mit dem Computer wird als Clinical-DecisionSupport-System (CDSS) bezeichnet. Solche Bestrebungen und die teilweise vorhandene Aversion der Ärzteschaft dagegen gibt es schon seit mehr als 20 Jahren. Keine Angst: Die Software wird und darf uns nie die Entscheidung abnehmen. Sie kann auch keine Verantwortung übernehmen.

Aber: Wieso sollen die wichtigsten verfügbaren Informationen zum Zeitpunkt der Entscheidung nicht miteinbezogen werden? Damit dies möglich ist, sollten diese Informationen höchstens einen Klick entfernt sein. Die folgenden zwei Definitionen umschreiben, was unter CDSS zu verstehen ist:

- «Clinical Decision Support Systems link health observations with health knowledge to influence health choices by clinicians for improved health care» [9].

- «Clinical DSSs are typically designed to integrate a medical knowledge base, patient data and an inference engine to generate casespecific advice» [10].

Die CDSS-Tools sollen uns zum Zeitpunkt der Entscheidung die besten derzeit verfügbaren Informationen zur Verfügung stellen. CDSS sollen uns in der Entscheidungsfindung unterstützen. Wir alle sind bestrebt, die für die aktuelle Situation beste Entscheidung zu treffen. Diese «beste» Entscheidung kann je nach verfügbaren Informationen aber eben unterschiedlich ausfallen. Das Zur-Verfügung-Stellen von Information ist die Kernkompetenz der Informationstechnologie. Dies dürfen wir neidlos anerkennen und davon profitieren.

Künftige Systeme sollen auch eine minimale Intelligenz enthalten; die Implementierung solcher Tools in den klinischen Alltag ist aber noch weiter entfernt.

Weiterführende Referenzen dazu sind im Literaturverzeichnis erwähnt.

\section{Sinnvolle Klassifizierung}

Der Klassifizierung kommt in Zukunft eine zentrale Bedeutung zu: Dokumentation ist gut, klassifizierte Dokumentation ist besser. ICPC-2 ist die derzeit einzige vernünftige Klassifikation für die Hausarztmedizin. Die SGAM hat kürzlich die nationale Lizenz erworben. Wichtig in diesem Zusammenhang zu wissen: Es muss nicht jeder alles codieren. Es genügt, wenn ein repräsentatives Netz von Kollegen diese Codierung macht. Nur so können wir Aussagen darüber 
machen, was wir tun. Dies wird in der politischen Diskussion von morgen unabdingbar sein.

Weshalb ICPC-2 von der WONCA, der SGAM und in vielen Ländern in der Grundversorgung favorisiert wird, zeigt folgendes Beispiel: Patientin A hat eine Tante, die nach kurzer Krankheit an einem Hirntumor verstorben ist. Sie hatte von der Verwandtschaft gehört, dass die Tante intensive Kopfschmerzen hatte, bevor sie zum Arzt ging. Patientin A hat schon jahrelange, teilweise intensivste Kopfschmerzen. Am besten hilft Ibuprofen, Paracetamol hilft wenig, Migränemittel helfen gar nicht. Sie meldet sich, da sie Angst hat, ebenfalls einen Hirntumor zu haben.

In ICPC-2 können die Angst vor Hirntumor (N26), der chronische Kopfschmerz (N95) und sogar allenfalls diesbezüglich veranlasste Untersuchungen kodiert werden. Auch das Ergebnis «keine Erkrankung» (A97) ist mit dem Code erfassbar. Mit einer Abfolge solcher ICPC-2Codes auf der Zeitachse kann unsere Arbeit vollumfänglich dokumentiert werden. Sinnvoll ist eine solche Codierung nur mit elektronischer Unterstützung in Form eines Thesaurus und von Auswahlmöglichkeiten.

\section{Kritik an heutigen Systemen}

\section{Demoversion}

Praktisch jede kommerzielle Software hat ein Demonstrations- oder Evaluationspaket. Weshalb dies bei den heute führenden Praxisinformationssystemen nicht möglich sein sollte, ist nicht nachvollziehbar. Das Open-Source-Produkt «Elexis» (www.elexis.ch) ist hier eine löbliche Ausnahme.

\section{Datenmigration}

Bisher hat noch keine Softwarefirma Anstrengungen unternommen, damit Daten einfach aus einem System exportierbar und in einer zweiten elektronischen Krankengeschichte importierbar sind. Die Daten gehören uns Ärzten. Es kann doch nicht sein, dass ich, nur weil ich den Kleiderschrank bei einem Anbieter gekauft habe, wohl Wäsche einlagern und bei Bedarf ansehen kann, gleichzeitig aber nicht in der Lage bin, die ganze Kollektion in einen anderen Schrank zu zügeln. Es muss ja nicht gerade der Anbieterwechsel im Visier sein. Schon dass Patient A umzieht und seine Akten in elektronischer Form dem neuen Hausarzt übergeben möchte, sollte Anlass genug sein, hier Abhilfe zu schaffen. Auch in dieser Hinsicht konnte mit Unterstützung der Arbeitsgruppe SGAM. Informatics ein erstes Projekt realisiert werden, bezeichnenderweise wieder mit Elexis [11].

\section{Anforderungen}

Somit ergeben sich folgende Anforderungen an ein System der Zukunft:

- elektronische Krankengeschichte als zentrales Element;

- strukturierte, datenbankbasierte Dokumentation;

- Geräteschnittstellen;

- Demoversion verfügbar;

- plattformunabhängig;

- modularer Aufbau, moderne Softwarearchitektur, einfache Erweiterbarkeit;

- Integration von CDSS;

- Export und Importfunktion;

- Implementierung von ICPC-2.

Obwohl sich in den letzten Jahren einiges getan hat, sind die Bedürfnisse, die der klinische Praxisalltag mit sich bringt, noch bei weitem nicht umgesetzt. Die Softwarelandschaft der Arztpraxis wird sich verändern. Nur wer die Bedürfnisse der Anwender umsetzt, kann überleben.

Am 13. September 2007 findet in Olten ein erster Workshop «Evaluation elektronische Krankengeschichte» statt. Weitere Infos unter www.sgam.ch $\rightarrow$ Aktuell.

\section{Literatur}

1 Das Praxisinformationssystem der Zukunft: Vom PAS zum PIS. Softwarekatalog FMH Services; 2007.

2 E-Prescribing. Online-Abhandlung unter www. openclinical.org, dort u.a. Miller RA, et al. Clinical decision support and electronic prescribing systems: a time for responsible thought and action. J Am Med Inform Assoc. 2005;12(4):403-9.

3 Implementing the electronic medical record in the exam room: the effect on physician-patient communication and patient satisfaction. The Permanente Journal. 2007;2:21

4 Schattner P. Computing and information management in general practice. New York: McGraw-Hill; 2007.

5 Weitere Abfragemöglichkeiten siehe CD-ROM «Die elektronische Krankengeschichte». SGAM. Informatics. 2. Auflage. 2006.

6 Zoller M. Forschung bei Hausärzten - auf Knopfdruck möglich? PrimaryCare. 2006;6(4):71-2.

7 Übersicht der Module einzelner Hersteller unter www.fmhservices.ch $\rightarrow$ Software $2007 \rightarrow$ Übersicht weitere Module.

8 Z.B. Selectra Junior von Vitalab (Schweizer Vertretung Axonlab).

9 Dr. Robert Hayward vom Centre for Health Evidence. http://en.wikipedia.org/wiki/Clinical_ decision_support_system.

10 www.openclinicals.org.

11 XChange. PrimaryCare. 2007;7(16):267-70. 


\section{Weiterführende Literatur}

- Berner ES. Clinical Decision Support Systems. Health Informatics Series. $2^{\text {nd }}$ edition. Heidelberg: Springer; 2007.

- Greenes RA. Clinical Decision Support. Oxford: Elsevier; 2007.

- Miller RH, Sim I. Physicians' use of electronic medical records: barriers and solutions clinical decision support in electronic prescribing: recommendations and an action plan. Report of the Joint Clinical Decision Support Workgroup. March 2005

- Summary of a Workshop on developing a roadmap for national action on clinical decision support, Washington D.C. October 26-27, 2005.

- Kuo GM, et al. Cross-sectional comparison of electronic and paper medical records on medication counseling in primary care clinics. J Am Board Fam Med. 2007;20(2):164-73.

- Wang SJ. A cost-benefit analysis of electronic medical records in primary care 2003. Experta Medica Inc.
- Garets D. Electronic medical records vs. electronic health records: Yes, there is a difference. HIMSS Analytics, LLC. January 26, 2006.

- Garg AX, et al. Effects of computerized clinical decision support systems on practitioner performance and patient outcomes. A systematic review. JAMA. 2005;293(10):1223-38.

- Welch WP. Electronic health records in four community physician practices: impact on quality and cost of care. American Medical Informatics Association; 2007.

- Crosson JC. Electronic medical records and diabetes quality of care: results from a sample of family medicine practices. Ann Fam Med. 2007;5:209-15.

- eHealth 101: Electronic medical records reduce costs, improve care, and save lives. American Electronics Association; 2006. 